\title{
Etiologia dos transtornos alimentares: aspectos biológicos, psicológicos e sócio-culturais
} Etiology of eating disorders: biological,
psychological and sociocultural
determinants Christina M Morgana, Ilka Ramalho Vecchiattia e André Brooking Negrãob

aPrograma de Atendimento aos Transtornos Alimentares, Departamento de Psiquiatria, Universidade Federal de São Paulo. São Paulo, SP, Brasil. ${ }^{\text {b} A m b u l a t o ́ r i o ~ d e ~ B u l i m i a ~ e ~ T r a n s t o r n o s ~ A l i m e n t a r e s ~(A m b u l i m), ~ I n s t i t u t o ~ d e ~ P s i q u i a t r i a ~ d o ~ H o s p i t a l ~ d a s ~ C l i ́ n i c a s, ~ F a c u l d a d e ~ d e ~ M e d i c i n a ~ d a ~ U n i v e r-~}$ sidade de São Paulo. São Paulo, SP, Brasil

Resumo Os transtornos alimentares possuem uma etiologia multifatorial, composta de predisposições genéticas, socioculturais e vulnerabilidades biológicas e psicológicas. Entre os fatores predisponentes, destacam-se a história de transtorno alimentar e (ou) transtorno do humor na família, os padrões de interação presentes no ambiente familiar, o contexto sociocultural, caracterizado pela extrema valorização do corpo magro, disfunções no metabolismo das monoaminas centrais e traços de personalidade. A dieta é o comportamento precursor que geralmente antecede a instalação de um transtorno alimentar. Contudo, a presença isolada da dieta não é suficiente para desencadear o transtorno alimentar, tornando-se necessária uma interação entre os fatores de risco e outros eventos precipitantes. Por último, o curso transitório ou crônico de um transtorno alimentar está relacionado à persistência de distorções cognitivas, à ocorrência de eventos vitais significativos e a alterações secundárias ao estado de desnutrição.

Descritores Família. Sociedade. Personalidade. Anorexia nervosa. Bulimia nervosa.

Abstract Eating disorders have a multifactorial etiology, composed by genetic predisposition, sociocultural factors, and biological and psychological vulnerabilities. Among the predisposing factors, emphasis is given to a history of eating and/or mood disorders, personality traits, patterns of family interaction, alterations in brain monoamines and sociocultural overvalued drive for thinness. Dieting is the most frequently event present at the onset of an eating disorder. Nevertheless, dieting itself is not sufficient to precipitate an eating disorder. An interaction among risk factors and other precipitating events is necessary. Finally, the chronic or transitory course of an eating disorder is related to the persistence of distorted cognitions, to the occurrence of significant vital events, and to starvation-induced psychobiological alterations.

Keywords Family. Society. Personality. Anorexia nervosa. Bulimia nervosa.

\section{Introdução}

Os transtornos alimentares (TA) têm uma etiologia multifatorial, ou seja, são determinados por uma diversidade de fatores que interagem entre si de modo complexo, para produzir e, muitas vezes, perpetuar a doença. Classicamente, distinguem-se os fatores predisponentes, precipitantes e os mantenedores dos TA. ${ }^{1}$ Os fatores predisponentes são aqueles que aumentam a chance de aparecimento do TA, mas não o tornam inevitável. Os fatores que precipitam a doença marcam o aparecimento dos sintomas dos TA. Finalmente, os fatores mantenedores determinam se o transtorno vai ser perpetuado ou não.

\section{Fatores predisponentes}

Estudos caso-controle ${ }^{2,3}$ realizados na comunidade, revelaram que há duas classes de fatores de risco para os TA: uma inclui o risco para transtornos psiquiátricos em geral e outra é 
Tabela 1 - Fatores predisponentes.

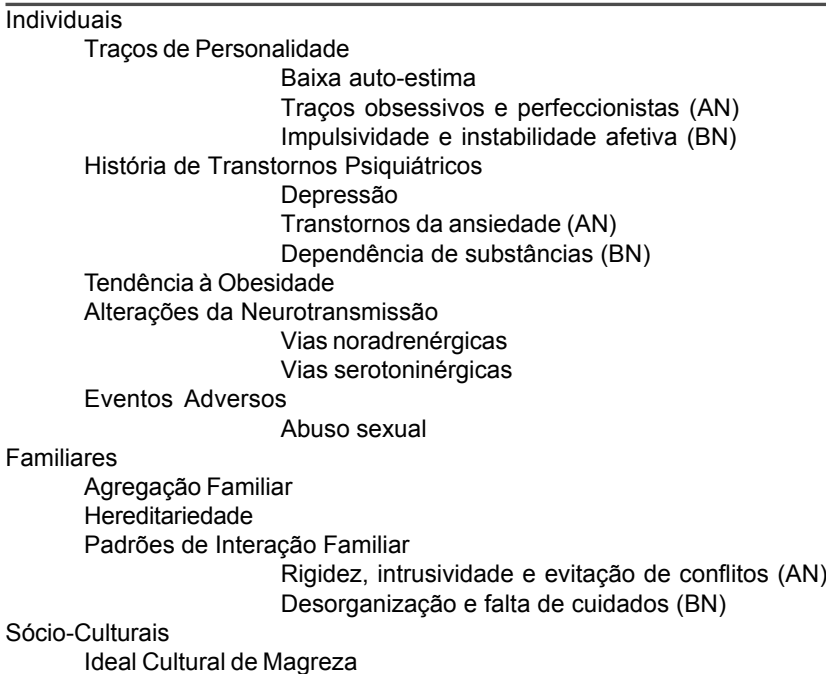

específica para os TA. Os fatores da primeira classe incluem a comorbidade com outras patologias psiquiátricas, a história de transtornos psiquiátricos na família, abuso sexual ou físico e adversidades na infância. Os fatores mais específicos incluem os traços de personalidade, o risco para desenvolvimento da obesidade e a realização de uma dieta calórica restritiva. Os fatores predisponentes dos TA são categorizados em três grupos: individual, familiar/hereditário e sociocultural.

\section{Fatores individuais}

\section{Personalidade e outros transtornos psiquiátricos}

Traços como obsessividade, perfeccionismo, passividade e introversão são comuns em pacientes com anorexia nervosa (AN) e permanecem estáveis mesmo após a recuperação do peso. ${ }^{4,5}$ As características de personalidade da bulimia nervosa (BN) são diferentes: sociabilidade, comportamento gregário, comportamentos de risco e impulsividade. Estes traços são consistentes com o descontrole e a purgação. ${ }^{6} \mathrm{~A}$ impulsividade e a instabilidade afetiva parecem ser aspectos centrais do temperamento de indivíduos com BN, ${ }^{7}$ especialmente aqueles que apresentam dependência química. ${ }^{8}$ Estudos recentes têm sugerido que a baixa auto-estima ${ }^{9,10}$ ou auto-avaliação negativa ${ }^{3,11}$ são fatores de risco importantes tanto para $\mathrm{AN}$ quanto para $\mathrm{BN}$.

Há uma associação entre a $\mathrm{AN}$ e transtornos da personalidade obsessiva-compulsiva, da BN, com transtornos caracterizados pela impulsividade e instabilidade, e do transtorno da compulsão alimentar periódica (TCAP), com transtornos do tipo evitativo e ansioso. ${ }^{12} \mathrm{~A}$ história pré-mórbida de transtorno psiquiátrico, especialmente depressão, também é um fator de risco para os TA, particularmente para a BN. ${ }^{11} \mathrm{~A}$ AN parece estar associada a transtornos da ansiedade, ${ }^{5}$ enquanto a BN também ocorre com freqüência em indivíduos com dependências químicas. ${ }^{8}$ Crianças obesas que apresentam episódios de compulsão alimentar têm maiores índices de ansiedade e depressão quando comparadas a crianças obesas sem perda de controle alimentar. ${ }^{13}$

\section{Somáticos}

Algumas pesquisas, ${ }^{3,14}$ mas não todas,${ }^{15}$ sugerem que a puberdade precoce é um fator de risco para os TA, particularmente para a BN. O aumento importante da gordura corporal em meninas adolescentes requer uma reorganização da imagem corporal e pode reforçar as preocupações com o peso. ${ }^{16}$

A tendência à obesidade parece estar associada aos $\mathrm{TA}^{5}$, algo que, na verdade, parece ser mediado por uma maior tendência a fazer dieta. ${ }^{1}$ A obesidade também prediz um aumento das brincadeiras relacionadas ao peso, ${ }^{17}$ aumentando a pressão social para emagrecer. Conseqüentemente, a obesidade pode também ter efeitos deletérios na auto-estima e na satisfação corporal, especialmente em adolescentes com auto-imagem negativa, vulneráveis às pressões culturais pela magreza.

Surpreendentemente, os estudos sobre os fatores de risco para a $\mathrm{BN},{ }^{11}$ a AN, ${ }^{3}$ e o TCAP ${ }^{2}$ indicam que a tendência à obesidade e à dieta, embora relevantes para a etiologia da $\mathrm{AN}$ e do TCAP, são mais importantes no desenvolvimento da BN. Tais achados sugerem que pessoas que desenvolvem $\mathrm{BN}$ são mais pesadas, o que pode torná-las mais sensíveis à sua aparência física e, assim, mais propensas a iniciar uma dieta. ${ }^{3}$

Alterações em vias noradrenérgicas e da serotonina (5-HT) podem exercer seu papel predisponente por meio de ações primariamente no humor, controle do impulso, obsessividade e regulação de fome e saciedade nos TA. ${ }^{18}$ Inexiste um consenso se as alterações encontradas nos valores de noradrenalina e seus metabólitos na AN indicam aumento ou diminuição da atividade da noradrenalina nesta doença. ${ }^{19-21} \mathrm{~A}$ mesma indefinição existe para a 5-HT e seus metabólitos na AN. As concentrações séricas de 5-HT encontraram-se elevadas. Contudo, o maior metabólito da 5-HT encontra-se diminuído em pacientes com $\mathrm{AN} .{ }^{22-24}$ É bem reconhecido que a administração de agentes serotoninérgicos em animais e pessoas sem transtornos alimentares suprime o comportamento alimentar (velocidade do ato de se alimentar e tamanho das refeições), particularmente, diminuindo a ingestão de carboidratos. ${ }^{25}$ Esses efeitos da 5-HT podem ser resumidos como uma intensificação da saciedade após o inicio do comportamento consumatório de alimento. Uma vez que existe uma clara preferência por alimentos altamente calóricos, marcadamente carboidratos, durante os episódios de compulsão alimentar, tal comportamento pode ser visto como uma alteração na produção da saciedade, mais especificamente, por uma atividade deficiente na circuitaria serotoninérgica dos pacientes com BN. ${ }^{26}$ Várias linhas de evidências corroboram esta hipótese: diminuição da responsividade do receptor pós-sináptico de serotonina, achatamento da produção de prolactina após estimulação por agentes serotoninérgicos e controle parcial dos episódios bulímicos por agentes que facilitam a transmissão serotoninérgica. ${ }^{22,27,28}$ Assim, um fator predisponente no desenvolvimento da BN seria a menor atividade de sistemas associados à 5 -HT cerebral.

\section{Eventos vitais adversos}

Diversos estudos já sugeriram que experiências sexuais adversas podem ser fatores importantes na etiologia dos TA.,29 Hoje em dia, considera-se que o trauma sexual aumenta a 
vulnerabilidade a transtornos psiquiátricos em geral, ${ }^{11,30}$ sendo assim um fator de risco não-específico, particularmente, para a $\mathrm{BN}$ e, em menor grau, para a AN. ${ }^{31}$

\section{Fatores na família e hereditários Agregação familiar}

Há, claramente, um padrão de agregação dos TA nas famílias de indivíduos diagnosticados com AN e BN. Ou seja, tais TA são mais freqüentes em parentes de primeiro grau dos pacientes com TA do que em parentes de primeiro grau de indivíduos saudáveis. ${ }^{32,33}$ Parentes em primeiro grau de indivíduos com AN apresentam uma chance 11 vezes maior de desenvolver o transtorno do que parentes de indivíduos saudáveis. ${ }^{34}$ Para a $\mathrm{BN}$, o risco é quatro vezes maior. ${ }^{34}$ Outros transtornos psiquiátricos também são freqüentes em parentes de primeiro grau de indivíduos com TA, ${ }^{8}$ embora apenas o transtorno de personalidade obsessivo-compulsivo e a fobia social pareçam ter uma vulnerabilidade familiar comum com a AN. ${ }^{8}$

Os estudos de agregação familiar dos TA implicam que há mecanismos de transmissão da doença dentro das famílias. Fatores genéticos (vide abaixo) têm sua importância, embora existam evidências demonstrando a participação de fatores ambientais específicos na transmissão da doença. Um estudo longitudinal, que avaliou recém-nascidos e seus pais no que diz respeito aos fatores preditivos de problemas alimentares na infância ${ }^{35}$ demonstrou que a insatisfação corporal da mãe, a internalização do ideal de magreza, o comportamento de fazer dieta, os sintomas bulímicos e maior índice de massa corporal da mãe e do pai aumentam a chance de aparecimento de problemas alimentares na infância, o que pode aumentar o risco para TA no futuro.

\section{Genética}

Evidências da contribuição de fatores genéticos provêm de estudos com gêmeos. A partir das diferenças de concordância da doença em pares de gêmeos monozigóticos e dizigóticos, é possível estimar a magnitude da influência de aspectos genéticos nos TA. Bulik et $\mathrm{al}^{36}$ publicaram a mais extensa revisão sobre o assunto e, mais do que isso, analisaram dados de estudos anteriores usando uma metodologia única e amplamente aceita. Os autores concluem que, seguramente, há uma contribuição genética na bulimia nervosa, com valores entre $31 \%$ a $83 \%$, tanto em amostras populacionais como em amostras clínicas. ${ }^{36}$ Embora alguns estudos apontem para um papel que varia de $0 \%$ a $80 \%$ de participação de aspectos genéticos na etiologia da $\mathrm{AN}$, o mesmo estudo de Bulik et al $^{36}$ afirma que ainda não é possível estimar o real peso de aspectos genéticos na etiologia da doença. Isto porque, além da prevalência baixa da $\mathrm{AN}$, dado que reduz o poder dos testes estatísticos nestes estudos, eles são poucos e incorrem em erros metodológicos comuns em estudos de gemelares. ${ }^{36,37}$

\section{Padrões de interação familiar}

Do ponto de vista subjetivo, indivíduos com AN consideram suas famílias estáveis, sem conflitos, coesas e não se queixam de falta de cuidados ou atenção. ${ }^{38}$ Já observadores externos notam que, quando comparados a famílias de indivíduos normais, as famílias dos anoréxicos são mais rígidas, intrusivas e tendem a evitar os conflitos. ${ }^{38}$ Já as pacientes com BN descrevem as famílias como mais perturbadas, mal organizadas e se queixam da falta de afeto e cuidados, características confirmadas pela observação externa. ${ }^{6}$ Contudo, não é certo se tais características estão presentes antes do estabelecimento do transtorno. ${ }^{6}$ Uma relação distante e pouco afetiva com o pai também parece estar prospectivamente associada a dificuldades alimentares na adolescência. ${ }^{39}$

\section{Fatores sócio-culturais}

O ideal de beleza feminina centrado na magreza é parte integrante da psicopatologia dos TA. Na cultura ocidental, ser magra significa ter competência, sucesso, autocontrole e ser atraente sexualmente. ${ }^{40}$ Vindo ao encontro destes valores, as dietas restritivas e cirurgias plásticas transmitem a ilusão de que o corpo é infinitamente maleável. Uma vez que o ideal de magreza proposto é uma impossibilidade biológica para a maioria das mulheres, a insatisfação corporal tem se tornado cada vez mais comum. ${ }^{41}$ Assim sendo, o contexto sociocultural e os fatores que modulam seu impacto em cada indivíduo têm lugar assegurado na gênese dos TA. ${ }^{40}$

O padrão de beleza veiculado pelos meios de comunicação e pelo convívio social parece exercer um efeito marcante sobre as mulheres. Universitárias sem TA expostas ao modelo de corpo magro, a imagens neutras e a imagens de mulheres de peso normal/sobrepeso relataram que as mulheres magras eram tidas como mais atraentes e que a exposição ao modelo magro gerava um aumento de respostas afetivas negativas (culpa, depressão, infelicidade, vergonha). ${ }^{40}$ Mais ainda, o reforço social exercido pela família, pelos amigos e pela mídia em adolescentes e adultas jovens para se ter o corpo magro relaciona-se à presença de sintomas bulímicos e prediz o início de sintomas nesta população. ${ }^{42}$

O papel das mães na formação da opinião de seus filhos quanto ao valor da aparência, do peso e da forma corporal também foi investigado. Mães de pacientes com TA tendem a ser mais críticas e preocupadas com relação ao peso de suas filhas, incentivando-as a fazer dieta mais do que as mães de filhas sem TA. ${ }^{16}$ Ricciardelli \& $\mathrm{McCabe}^{43}$ afirmam que a pressão para perder peso exercida pela mãe é o principal fator preditivo de insatisfação corporal e do engajamento em estratégias para modificar o corpo em adolescentes de ambos os sexos.

Tradicionalmente, acreditava-se que os TA limitavam-se a um grupo constituído por mulheres jovens, brancas, pertencentes à elite e residentes em países ricos, algo que vem sendo contestado pelo número crescente de relatos de TA em países em desenvolvimento e em diferentes etnias. ${ }^{44,45}$ Isto exemplifica, mais uma vez, o papel da mídia e da urbanização na predisposição aos TA. ${ }^{46}$ De fato, um maior grau de urbanização parece aumentar a chance de TA, sendo o grau de urbanização mais relacionado à $\mathrm{BN}$ do que à $\mathrm{AN} .{ }^{47} \mathrm{~A}$ urbanização levaria a uma maior exposição ao ideal de magreza através da mídia além de propiciar mudanças de hábitos alimentares (ex., "fast-foods"), sedentarismo e um maior número de pessoas 
com sobrepeso e obesidade.

Pertencer a grupos profissionais como atletas, bailarinas, modelos e nutricionistas reforçam a demanda por um corpo muito magro, aumentando o risco de TA..$^{48}$ Não está esclarecido ainda se o ambiente teria uma influência como desencadeante ou se pessoas já predispostas a desenvolver TA tenderiam a procurar tais profissões.

\section{Fatores precipitantes Dieta}

A dieta para emagrecer é o fator precipitante mais freqüente nos TA, sendo um comportamento comum nas sociedades ocidentais ou ocidentalizadas, onde predomina o ideal da magreza. Estudos longitudinais demonstram claramente que a dieta aumenta de modo considerável o risco para os TA. Indivíduos que faziam dieta tiveram um risco 18 vezes maior para o desenvolvimento de TA do que entre indivíduos que não faziam dieta após um ano de seguimento. ${ }^{49}$

A dieta, isoladamente, não é suficiente para produzir os TA. Ela precisa interagir com os fatores de risco já descritos para levar a este desfecho. ${ }^{50}$ Embora não seja possível especificar como os fatores de risco se combinam para gerar a $\mathrm{AN}$ em alguns indivíduos e a $\mathrm{BN}$ em outros, o desenvolvimento da $\mathrm{BN}$ parece estar associado também a uma propensão para a obesidade e para dificuldades no controle dos impulsos..$^{50}$ Outra possibilidade é que a restrição alimentar favorece o aparecimento das compulsões alimentares, o que inicia o ciclo compulsão/ purgação da BN. Algumas pessoas em restrição alimentar, no entanto, conseguem aumentar cada vez mais a restrição sem ter compulsão. Assim, instala-se a desnutrição, que aumenta a distorção da imagem corporal e, conseqüentemente, aumenta também o medo de engordar e o desejo de emagrecer, perpetuando assim a AN. ${ }^{16}$

\section{Eventos estressores}

Tanto a AN quanto a BN parecem ser precedidas por uma maior freqüência de eventos estressores quando comparadas a controles normais. ${ }^{51}$ Eventos envolvendo uma desorganização da vida ou uma ameaça à integridade física (doença, gravidez, abuso sexual e físico) são mais freqüentes em indivíduos com $\mathrm{BN}$ do que em controles e podem ter um papel desencadeador do transtorno por reforçar sentimentos de insegurança e inadequação. ${ }^{51} \mathrm{O}$ impacto que os eventos estressores podem ter sobre a patogênese dos TA depende dos recursos que cada indivíduo possui para responder aos mesmos. Assim, pacientes com anorexia nervosa tendem a ter uma atitude de evitação diante de uma crise, enquanto pacientes com bulimia nervosa apresentam mais ruminações cognitivas.

\section{Fatores mantenedores}

Por último, o estudo dos fatores mantenedores da doença inclui o papel das alterações fisiológicas e psicológicas produzidas pela desnutrição e pelos constantes episódios de compulsão alimentar e purgação, que tendem a perpetuar o transtorno. Muitas vezes, os fatores que mantêm o problema alimentar são diferentes daqueles que foram responsáveis pelo seu desenvolvimento inicial. A privação alimentar leva, sabidamente, a alterações físicas e psicológicas, sendo que muitas características dos pacientes com transtorno alimentar são resultado e não causa - do ciclo vicioso desencadeado pela desnutrição. Por exemplo, já está bem estabelecido atualmente o papel da restrição alimentar no desencadeamento de episódios de compulsão alimentar, ou ainda, na ocorrência de pensamentos obsessivos sobre comida, que reforçam a necessidade inicial de controle.

$\mathrm{Na} \mathrm{AN}$, o estado de desnutrição gera alterações neuroendócrinas que podem contribuir para a manutenção de vários dos sintomas da doença. Pacientes com AN demonstraram valores séricos aumentados de cortisol ${ }^{52,53}$ bem como evidências indiretas de aumento da atividade do hormônio liberador de corticotropina (CRH) ${ }^{54,55}$ De modo análogo, experimentos clássicos de privação alimentar acarretam um aumento da produção de cortisol através de uma maior liberação de CRH. ${ }^{56}$ As alterações de CRH e cortisol na AN são estado-dependentes. A recuperação parcial ou total de peso dos pacientes reverte o quadro de valores elevados de cortisol, indicando que esta anormalidade é secundária ao quadro de inanição. ${ }^{57,58}$ Levando-se em conta que o $\mathrm{CRH}$, quando injetado no sistema nervoso central de modelos animais, causa anorexia, aumenta a atividade motora e inibe a atividade sexual, postula-se que, uma vez atingida uma perda importante de peso, sintomas como a anorexia, perda da libido e hiperatividade vistos na AN sejam reforçados por uma maior atividade de CRH no cérebro. ${ }^{54}$ Desta maneira, uma possível hiperatividade de $\mathrm{CRH}$ pode contribuir para manter o círculo vicioso da perpetuação de um baixo peso na AN já instalada.

Alterações dos níveis plasmáticos de leptina refletem mais um distúrbio hormonal secundário ao estado de desnutrição e da perda de peso na AN. Em indivíduos normais, a leptina veicula um sinal de saciedade para o cérebro e seus valores séricos estão diretamente correlacionados com o peso e, mais precisamente, refletem a massa de gordura do indivíduo. ${ }^{59}$ Vários estudos demonstraram que a correlação entre massa de gordura e leptina está preservada na AN, ou seja, os valores absolutos de medidas pontuais da leptina são baixos, durante a fase de perda de peso, e são maiores, quando da recuperação ponderal. ${ }^{60,61}$ Contudo, em dois estudos independentes foi observado que a leptina atingiu valores normais antes da recuperação integral do peso em pacientes com AN em tratamento. ${ }^{61,62} \mathrm{Tal}$ dado sugere que a normalização prematura da leptina pode dificultar a chegada e a manutenção de um peso normal na AN. Uma vez em tais níveis, a leptina estaria gerando um sinal errôneo de saciedade para o cérebro quando na verdade o peso corporal ainda não está integralmente restituído.

Episódios bulímicos experimentais induzidos em indivíduos normais têm um impacto importante sobre o metabolismo de carboidratos. ${ }^{63}$ Observou-se um aumento significativo da glicemia de jejum no dia seguinte a um episódio bulímico experimental, além de um aumento ainda maior da resposta de insulina durante o teste bulímico quando comparados aos valores obtidos durante três dias de refeições normais. Este estudo demonstra o impacto adverso de episódios bulímicos sobre o metabolismo da glico- 
se e insulina, e tais evidências possivelmente justificam a dificuldade de se estabelecer um balanço energético adequado em pacientes com BN e aqueles com TCAP. ${ }^{63}$

Fatores psicológicos, interpessoais e culturais também atuam perpetuando o transtorno. Não se pode esquecer, por exemplo, dos aspectos reforçadores produzidos pelo sucesso em controlar o peso - tão valorizados culturalmente - em indivíduos que costumam vivenciar sentimentos de baixa auto-estima e sensação de falta de controle sobre a própria vida. (Tabela 3)

\section{Conclusões}

A gama de aspectos envolvidos na etiologia dos TA é imensa. O desafio para aqueles que trabalham tanto na clínica como na pesquisa destes transtornos não é simplesmente descrever todos os elementos envolvidos, mas, sim, o de compreender
Tabela 3 - Fatores mantenedores.

Fisiológicos

Privação alimentar favorece episódios de compulsão alimentar Episódios de compulsão alimentar interferem no metabolismo da glicose e insulina

Psicológicos

Privação alimentar desencadeia pensamentos obsessivos sobre Culturais comida e maior necessidade de controle

Magreza vista como símbolo de sucesso

como diversos fatores interagem entre si em cada caso ou situação. Finalmente, é importante lembrar que os TA não emergem abruptamente, mas se desenvolvem ao longo de vários anos, a partir de predisposições presentes desde o nascimento do indivíduo, de vulnerabilidades que emergem nas primeiras etapas da vida e de ocorrências mais tardias na sua história. ${ }^{6}$

\section{Referências}

1. Cooper Z. The development and maintenance of eating disorders. In: Brownell KD, Fairburn CG, editors. Eating disorders and obesity: a comprehensive handbook. New York: The Guilford Press; 1995. p. 199-206.

2. Fairburn CG, Doll HA, Welch SL, Hay PJ, Davies BA, O'Connor ME. Risk factors for binge eating disorder: a community-based, case-control study. Arch Gen Psychiatry 1998;55(5):425-32.

3. Fairburn CG, Cooper Z, Doll HA, Welch SL. Risk factors for anorexia nervosa: three integrated case-control comparisons. Arch Gen Psychiatry 1999;56(5):468-76.

4. Srinivasagam NM, Kaye WH, Plotnicov KH, Greeno C, Weltzin TE, Rao R. Persistent perfectionism, symmetry, and exactness after long-term recovery from anorexia nervosa. Am J Psychiatry 1995;152(11):1630-4.

5. Rastam M. Anorexia nervosa in 51 Swedish adolescents: premorbid problems and comorbidity. J Am Acad Child Adolesc Psychiatry 1992;31(5):819-29.

6. Lask B. Aetiology. In: Lask B, Bryant-Waugh R, editors. Anorexia nervosa and related eating disorders in childhood and adolescence. 2nd ed. East Sussex: Psychology Press; 2000. p. 63-79.

7. Diaz-Marsa M, Carrasco JL, Saiz J. A study of temperament and personality in anorexia and bulimia nervosa. J Personal Disord 2000;14(4):352-9.

8. Lilenfeld LR, Kaye WH, Greeno CG, Merikangas KR, Plotnicov $\mathrm{K}$, Pollice $\mathrm{C}$, et al. A controlled family study of anorexia nervosa and bulimia nervosa: psychiatric disorders in first-degree relatives and effects of proband comorbidity. Arch Gen Psychiatry 1998;55(7):603-10.

9. McGee R, Williams S. Does low self-esteem predict health compromising behaviours among adolescents? J Adolesc 2000;23(5):569-82.

10. Ghaderi A, Scott B. Prevalence, incidence and prospective risk factors for eating disorders. Acta Psychiatr Scand 2001;104(2):122-30.

11. Fairburn CG, Welch SL, Doll HA, Davies BA, O'Connor ME. Risk factors for bulimia nervosa. A community-based case-control study. Arch Gen Psychiatry 1997;54(6):509-17.

12. Grilo CM. Recent research of relationships among eating disorders and personality disorders. Curr Psychiatry Rep 2002;4(1):18-24.
13. Morgan CM, Yanovski SZ, Nguyen TT, McDuffie J, Sebring NG, Jorge MR, et al. Loss of control over eating, adiposity, and psychopathology in overweight children. Int J Eat Disord 2002;31(4):430-41.

14. Kaltiala-Heino R, Rimpela M, Rissanen A, Rantanen P. Early puberty and early sexual activity are associated with bulimictype eating pathology in middle adolescence. J Adolesc Health 2001;28(4):346-52.

15. Ackard DM, Peterson CB. Association between puberty and disordered eating, body image, and other psychological variables. Int J Eat Disord 2001;29(2):187-94.

16. Gowers SG, Shore A. Development of weight and shape concerns in the aetiology of eating disorders. Br J Psychiatry 2001;179:23642 .

17. Stice E, Agras WS, Hammer LD. Risk factors for the emergence of childhood eating disturbances: a five- year prospective study. Int J Eat Disord 1999;25(4):375-87.

18. Spoont MR. Modulatory role of serotonin in neural information processing: implications for human psychopathology. Psychol Bull 1992;112(2):330-50.

19. Gross HA, Lake CR, Ebert MH, Ziegler MG, Kopin IJ. Catecholamine metabolism in primary anorexia nervosa. J Clin Endocrinol Metab 1979;49(6):805-9.

20. Kaye WH, Ebert MH, Raleigh M, Lake R. Abnormalities in CNS monoamine metabolism in anorexia nervosa. Arch Gen Psychiatry 1984;41(4):350-5.

21. Van Binsbergen CJ, Odink J, Van der Beek EJ, Westenberg HM, Bennink HJ. Biogenic amines in anorexia nervosa: circadian rhythm in urinary excretion and influence of posture and physical task load on plasma catecholamines. Psychosom Med 1991;53(4):440-52.

22. Kaye WH. Persistent alterations in behavior and serotonin activity after recovery from anorexia and bulimia nervosa. Ann N Y Acad Sci 1997;817:162-78.

23. Gillberg C. Low dopamine and serotonin levels in anorexia nervosa. Am J Psychiatry 1983;140(7):948-9.

24. Hassanyeh F, Marshall EF. Measures of serotonin metabolism in anorexia nervosa. Acta Psychiatr Scand 1991;84(6):561-3.

25. Leibowitz SF, Alexander JT. Hypothalamic serotonin in control of eating behavior, meal size, and body weight. Biol Psychiatry 1998;44(9):851-64. 
26. Weltzin TE, Fernstrom MH, Kaye WH. Serotonin and bulimia nervosa. Nutr Rev 1994;52(12):399-408.

27. Jimerson DC, Lesem MD, Hegg AP, Brewerton TD. Serotonin in human eating disorders. Ann N Y Acad Sci 1990;600:532-44.

28. Bacaltchuk J, Hay P, Mari JJ. Antidepressants versus placebo for the treatment of bulimia nervosa: a systematic review. Cochrane Database Syst Rev 2000;2(2):310-7.

29. Pope HG-Jr, Mangweth B, Negrao AB, Hudson JI, Cordas TA. Childhood sexual abuse and bulimia nervosa: a comparison of American, Austrian, and Brazilian women. Am J Psychiatry 1994;151(5):732-7.

30. Palmer RL, Oppenheimer R. Childhood sexual experiences with adults: a comparison of women with eating disorders and those with other diagnoses. Int J Eating Disord 1992;12:359-64.

31. Wonderlich SA, Brewerton TD, Jocic Z, Dansky BS, Abbott DW. Relationship of childhood sexual abuse and eating disorders. J Am Acad Child Adolesc Psychiatry 1997;36(8):1107-15.

32. Hudson JI, Pope HG-Jr, Jonas JM, Yurgelun-Todd D, Frankenburg FR. A controlled family history study of bulimia. Psychol Med 1987; 17(4):883-90.

33. Strober M, Lampert C, Morrell W, Burroughs J, Jacobs C. A controlled family study of anorexia nervosa: evidence of familial aggregation and lack of shared transmission with affective disorders. Int J Eat Disord 1990;9:239-253.

34. Strober M, Freeman R, Lampert C, Diamond J, Kaye W. Controlled family study of anorexia nervosa and bulimia nervosa: evidence of shared liability and transmission of partial syndromes. Am J Psychiatry 2000;157(3):393-401.

35. Agras S, Hammer L, McNicholas F. A prospective study of the influence of eating-disordered mothers on their children. Int $\mathrm{J}$ Eat Disord 1999;25(3):253-62.

36. Bulik CM, Sullivan PF, Wade TD, Kendler KS. Twin studies of eating disorders: a review. Int J Eat Disord 2000;27(1):1-20.

37. Klump KL, Miller KB, Keel PK, McGue M, Iacono WG. Genetic and environmental influences on anorexia nervosa syndromes in a population-based twin sample. Psychol Med 2001;31(4):737-40.

38. Vandereycken W. The families of patients with an eating disorder. In: Brownell KD, Fairburn CG, editors. Eating disorders and obesity. 3 ed. New York: The Guilford Press; 1995. p. 219-23.

39. Johnson JG, Cohen P, Kasen S, Brook JS. Childhood adversities associated with risk for eating disorders or weight problems during adolescence or early adulthood. Am J Psychiatry 2002;159(3):394-400.

40. Stice E, Schupak-Neuberg E, Shaw HE, Stein RI. Relation of media exposure to eating disorder symptomatology: an examination of mediating mechanisms. J Abnorm Psychol 1994;103(4):836-40.

41. Pinhas L, Toner BB, Ali A, Garfinkel PE, Stuckless N. The effects of the ideal of female beauty on mood and body satisfaction. Int J Eat Disord 1999;25(2):223-6.

42. Stice E. Modeling of eating pathology and social reinforcement of the thin-ideal predict onset of bulimic symptoms. Behav Res Ther 1998;36(10):931-44.

43. Ricciardelli LA, McCabe MP. Children's body image concerns and eating disturbance: a review of the literature. Clin Psychol Rev 2001;21(3):325-44.

44. Nasser M. Screening for abnormal eating attitudes in a population of Egyptian secondary school girls. Soc Psychiatry Psychiatr Epidemiol 1994;29(1):25-30.
45. Negrao AB, Cordas TA. Clinical characteristics and course of anorexia nervosa in Latin America, a Brazilian sample. Psychiatry Res 1996;62(1):17-21.

46. Nasser M. Culture and weight consciousness. J Psychosom Res 1988;32(6):573-7.

47. Hoek HW, Bartelds AI, Bosveld JJ, van der Graaf Y, Limpens VE, Maiwald M, et al. Impact of urbanization on detection rates of eating disorders. Am J Psychiatry 1995;152(9):1272-8.

48. Sundgot-Borgen J. Risk and trigger factors for the development of eating disorders in female elite athletes. Med Sci Sports Exerc 1994;26(4):414-9.

49. Patton GC, Selzer R, Coffey C, Carlin JB, Wolfe R. Onset of adolescent eating disorders: population based cohort study over 3 years. BMJ 1999;318(7186):765-8.

50. Hsu LK. Can dieting cause an eating disorder? Psychol Med 1997;27(3):509-13

51. Welch SL, Doll HA, Fairburn CG. Life events and the onset of bulimia nervosa: a controlled study. Psychol Med 1997;27(3):515-22.

52. Baranowska B. Are disturbances in opioid and adrenergic systems involved in the hormonal dysfunction of anorexia nervosa? Psychoneuroendocrinology 1990;15(5-6):371-9.

53. Vierhapper H, Kiss A, Nowotny P, Wiesnagrotzki S, Monder C, Waldhausl W. Metabolism of cortisol in anorexia nervosa. Acta Endocrinol (Copenh) 1990;122(6):753-8.

54. Gold PW, Gwirtsman H, Avgerinos PC, Nieman LK, Gallucci WT, Kaye W, et al. Abnormal hypothalamic-pituitary-adrenal function in anorexia nervosa. Pathophysiologic mechanisms in underweight and weight-corrected patients. N Engl J Med 1986;314(21):1335-42.

55. Hotta M, Shibasaki T, Masuda A, Imaki T, Demura H, Ling N, et al. The responses of plasma adrenocorticotropin and cortisol to corticotropin-releasing hormone $(\mathrm{CRH})$ and cerebrospinal fluid immunoreactive $\mathrm{CRH}$ in anorexia nervosa patients. J Clin Endocrinol Metab 1986;62(2):319-24.

56. Timofeeva E, Richard D. Functional activation of CRH neurons and expression of the genes encoding $\mathrm{CRH}$ and its receptors in food-deprived lean $(\mathrm{Fa} /$ ?) and obese $(\mathrm{fa} / \mathrm{fa})$ Zucker rats. Neuroendocrinology 1997;66(5):327-40.

57. Doerr P, Fichter M, Pirke KM, Lund R. Relationship between weight gain and hypothalamic pituitary adrenal function in patients with anorexia nervosa. J Steroid Biochem 1980;13(5):529-37.

58. Fichter MM, Doerr P, Pirke KM, Lund R. Behavior, attitude, nutrition and endocrinology in anorexia nervosa. Acta Psychiatr Scand 1982;66(6):429-44.

59. Negrão AB, Licinio J. Leptina: o Diálogo entre Adipócitos e Neurônios. Arq Bras Endocrinol Metab 2000;44(3):205-14.

60. Hebebrand J, van der Heyden J, Devos R, Kopp W, Herpertz S, Remschmidt $\mathrm{H}$, et al. Plasma concentrations of obese protein in anorexia nervosa. Lancet 1995;346(8990):1624-5.

61. Mantzoros C, Flier JS, Lesem MD, Brewerton TD, Jimerson DC. Cerebrospinal fluid leptin in anorexia nervosa: correlation with nutrional status and potential role in resistance to weight gain. J Clin Endocrinol Metab 1997;82:1845-51.

62. Hebebrand J, Blum N, Coners H, Englaro P, Juul A. Leptin levels in patients with anorexia nervosa are reduced in the acute stage and elevated upon short-term weight restoration. Mol Psychiatry 1997;2:330-4.

63. Taylor AE, Hubbard J, Anderson EJ. Impact of binge eating on metabolic and leptin dynamics in normal young women. J Clin Endocrinol Metab 1999;84(2):428-34. 